multifocal dysplasia which resulted 3 colectomies. Four patients have been referred for consideration of liver transplantation.

Conclusions Robust surveillance in this group leads to timely diagnosis of malignancy and treatment. Establishment of integrated service for PSC-IBD patients results in early detection of complications, better compliance to guidelines and appropriate management of patients.

\section{PTU-112 DIAGNOSTIC ENQUIRY INTO UPPER GI AND HPB PATIENTS REFERRED ON THE URGENT SUSPECTED CANCER PATHWAY}

Rabia Lalani* , Jonathan Blackwell, Claire Woods, Deanne Bell, Debbie Kidanu, Megan Kell, James May, Sanjay Gupta. Department Of Gastroenterology and Medicine, Croydon University Hospital, London, UK

\subsection{6/gutjnl-2018-BSGAbstracts.490}

Introduction Patients on the Urgent Suspected Cancer (USC) pathway for gastroenterology and hepatology are referred for a wide range of conditions and abnormal results. NHS England aim to reduce the time to diagnosis to 28 days from the current 31 days (31 days to treat) by 2020 . Our aim was to determine the number and type of investigations performed on this pathway as a guide for services to plan for an increasing demand with lesser turnaround time.

Methods We performed a retrospective analysis of our USC database from April to December 2015. Patients were categorised into 3 groups based on the indication for referral -Group A was upper gastrointestinal (UGI) symptoms, Group B was hepato-pancreato-biliary (HPB) indications and Group C was all other non specific indications including abnormal tests and radiology.

Results A total of 911 patient records were reviewed. Of the 721 patients in Group A, 13 patients refused investigations $(\mathrm{n}=708) .436(60 \%)$ males, age 20-95 years (mean 64). In total, 1701 tests were done (mean $2.35 \pm 1$, median 2). Tests included 659 (93\%) gastroscopy (OGD); 367 ultrasound (US); 210 CT scans (30\%), 109 colonoscopies (15\%), 17 MRIs (2\%), 11 other radiology tests and 21 endoscopic tests. 102 patients $(14 \%)$ required 4 or more tests. In Group B $(n=95)$, age 2685 years (mean 65), males 41 (43\%), a total of 257 test were done (mean 2.7 \pm 1.5 , median 2) including 35 OGDs(37\%), 65 US (68\%), 59 CT scans (62\%), 35 MRIs (37\%), 10 ERCPs, 6 colonoscopies, 5 other radiology tests. 25 patients $(26 \%)$ required 4 or more tests. In Group $C n=95$, age 36-92 (mean 69), males 46 (48\%), 267 tests were done (mean 2.8 \pm 1.2 , median 3) including 77 OGDs (81\%), 37 colonoscopies (39\%), 29 US (31\%), 64 CT scans (67\%), 5 MRIs (5\%), 7 other radiology and 4 endoscopic tests. 28 patients (29\%) required 4 or more tests. There were 29 cancers detected in Group A(4\%), 23 (24\%) in Group B and 5 (5\%) in Group C.

Conclusions We have shown that a high proportion of patients with UGI symptoms and non specific symptoms need endoscopy while patients with HPB and other systemic symptoms require more cross sectional imaging. Diagnostic yield was highest for the HPB group. Overall more tests were required for HPB as compared to the UGI group and even more for the non-specific group. Between 25\%-30\% patients in HPB and non specific symptom group required 4 or more investigations. To reduce the time to diagnosis to 28 days by 2020 , both endoscopy and radiology will have to invest in infrastructure and manpower to avoid breeches especially for patients referred for HPB and non specific symptoms.

\section{PTU-113 IMPLEMENTING THE DECOMPENSATED CIRRHOSIS CARE BUNDLE IN A DISTRICT GENERAL HOSPITAL}

${ }^{1}$ Phey Shen Lee*, ${ }^{2}$ Andrea Broad, ${ }^{2}$ Allison Grapes, ${ }^{2}$ Jessica Pecqueur, ${ }^{2}$ Fraser Brown. ${ }^{1}$ South Tees Hospitals NHS Foundation Trust, Middlesbrough, UK ${ }^{2}$ Gateshead Health NHS Foundation Trust, Gateshead, UK

\subsection{6/gutjnl-2018-BSGAbstracts.491}

Introduction The Decompensated Cirrhosis Care Bundle was published by McPherson et al (BMJ 2014) in response to the 2013 National Confidential Enquiry into Patient Outcome and Death (NCEPOD) report into deaths from alcoholic liver disease. We describe our experience in implementing this at a district general hospital.

Methods The care bundle checklist was used as basis for audit pre-implementation in September 2014, post-implementation in June 2015 and re-audit in September-October 2016. Patients were identified via ICD codes, with review of electronic records and case notes.

Implementation The care bundle was made available on our trust intranet. We launched it with education sessions for medical and nursing staff in the Emergency Care Centre.

The AUDIT-C tool was embedded into the VitalPAC electronic observations system to ensure all patients were assessed for alcohol use. A 'Decompensated Cirrhosis' blood test panel was added to our electronic requesting system. Magnesium was added to calcium and phosphate under 'bone profile'.

Results Baseline results pre-implementation were comparable to regional data. Post-implementation results showed significant improvements which were sustained a year later (figures 1 and 2 ). $70 \%-80 \%$ of patients were reviewed by a gastroenterologist within 24 hours, improving from $44 \%$.

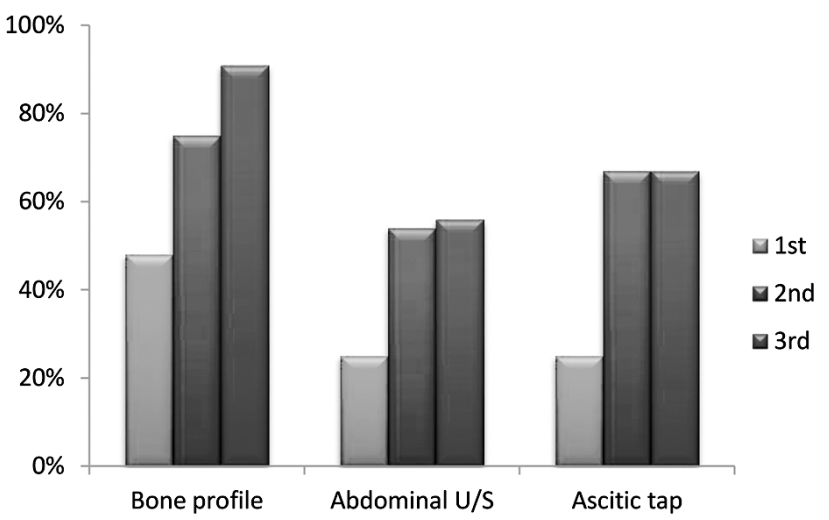

Abstract PTU-113 Figure 1 Basic investigations within first 6 hours of admission 